\title{
Analysis and Forecast of novel Coronavirus (Covid-19) Cases in India: A Simplified Mathematical Model based on Growth and Recovery
}

This paper was downloaded from TechRxiv (https://www.techrxiv.org).

\section{LICENSE}

CC BY 4.0

SUBMISSION DATE / POSTED DATE

$17-06-2020 / 17-06-2020$

\section{CITATION}

Rajalaxmi, R R; Aravind Vaithilingam, Chockalingam; Sivasubramanian, Gayathri; R, Lalitha (2020): Analysis and Forecast of novel Coronavirus (Covid-19) Cases in India: A Simplified Mathematical Model based on Growth and Recovery. TechRxiv. Preprint. https://doi.org/10.36227/techrxiv.12496535.v1

$\mathrm{DOI}$ 


\title{
Analysis and Forecast of novel Coronavirus (Covid-19) Cases in India: A Simplified Mathematical Model based on Growth and Recovery
}

\author{
R R Rajalaxmi \\ Professor \\ Computer Sciences and Engineering, Kongu Engineering College, Perundurai, India \\ *rrr@kongu.ac.in \\ Aravind CV \\ VERTICALS, High Impact Research Laboratories, Taylor's University Malaysia \\ aravindcv@ieee.org \\ Gayathri Sivasubramanian \\ Associate Professor, Management Studies, Vels Institute of Science Technology \& Advanced Studies, Chennai, \\ India \\ gayathri.sms@velsuniv.ac.in

\section{R Lalitha} \\ Professor, Computer Science and Engineering, Rajalakshmi Institute of Technology, Chennai, India \\ lalitha.r@ritchennai.edu.in \\ *Corresponding Author
}

\begin{abstract}
The 2019 novel coronavirus (Covid-19) outbreak has been treated as a pandemic by the World Health Organization. This work analyses the different phases of the Covid-19 outbreak in India and performs progress of the disease spread. The data is collected from Johns Hopkins epidemiological data providing the latest epidemiological data of from January 20, 2020 to May 31,2020. A simple mathematical model is developed to gather a quantitative picture of the epidemic spreading with limited reference data. Combining characteristics of the data from similar population density, we found part of the characteristics remain reasonably similar. Further, we performed an analysis and forecast of the disease spread in different phases of lockdown in the country. The profound model predictions considering the overall data exhibit that the numbers to reach peak between 28 August 2020 to 6 Sep 2020. As the pandemic still increases the number of infected cases, different quarantine levels would serve as an effective measure in containing the spread much earlier than the other similar cases. However, this depends on the real data pertaining to the disease spread for future cases.
\end{abstract}

\section{Keywords}

Pandemic, Covid-19, infection rate, mathematical model, recovery rate, quarantine level 


\subsection{Introduction}

Since mid of the last century several outbreaks are challenging humans through several new viruses and bacteria through various passive transmissions [1]. Today the world is witnessing the novel coronavirus (Covid-2019), the virus originated from the Severe Acute Respiratory Syndrome (SARS) family, that out broke earlier in South China in 2002-2003[2]. Due to its rapid transmission, both in patches and at larger community, the world health organization on $7^{\text {th }}$ Jan, 2020 announced this outbreak as pandemic after Spanish flu. It all started in Wuhan, on Dec 312019 as unknown pneumonia for 27 cases slowly transferred to become as an agent moving between the host populations [3]. Due to the nature of the virus behaviour on the host, the visibility is significant only the severity reaches $60 \%$, until then it looks as simple flu symptoms. This block of early detection makes the virus growth inside the host unnoticed. It is estimated that the virus can travel around 8 meters and can survive up to 3 hours on the surface [4].

The foremost criteria in the outbreak is the unavailability of the prediction system and tracing the infectious pathways, with most interests on the subsequent generation spreaders is quiet challenging. But, the outbreak of the 2019-CoV is just coincident with the large-scale population migration with the globalization impact throughout the world with six million people travel everyday through air, making the pandemic to grow exponentially within three months duration. In a way this has been a challenging aspect towards the epidemiological investigation, but simultaneously monitoring and prediction becomes highly meaningful for the decision making against the outbreak that created the public health crisis, which is attempted in this work, more in particular on the impact in India.

A pandemic with such intensity of spread as COVID 19 is a threat to the entire human race and a cornucopia of measures on various fronts are taken to contain the disease. Epidemiological modelling has a prominent role in predicting, assessing and containing such outbreaks. The 2019-nCoV data used in this study has data from the official website of Johns Hopkins University [5] providing the latest epidemiological data of global from January 20, 2020 up to the present. A lot of research labs and different organizations are collecting data about Covid19 spread globally. They also made it as open source so that researchers can make of the data to gain insights about the spread of the disease. The covid-19 dataset for different countries is collected from the GitHub repository (https://github.com/datasets/covid-19). It provides times series data from January 21, 2020 with the cumulative number of people affected by the coronavirus including:

- Number of people Confirmed with Coronavirus 
- Number of people Recovered from the infection

- Number of people died due to the deadly virus

The data is updated daily and available in CSV format so that anyone can download it for analysis.

The first SARS-CoV-2 virus stain infected person in India was identified on 29 Jan 2020, 19 days after its first observation in south china[6]. Reported cases remained relatively low until a large spike in cases in March 2020 as seen in Figure 1 [7]. Within the few days of reporting, the government put interests on the growing population of cases in relation to the size of the gathering at the national level. Since then the monitoring process is in place on the spread across the country breadth and width through measures at all the entry points into the country and within the country at several places. As the number was spiking, the Government announced lockdown on the 22 March 2020. The first step towards containment of outbreak started with the restricted movement in and out of the country from the 24 March 2020 for 21 days. At the end of first phase, the doubling rate reduced to eight days. As a precautionary measure the lock down was extended up to 3 May 2020 with conditional relaxation. Since the number of cases still raise, the government decided to prolong the lockdown up to 17 May 2020. Here, the country has been divided into 3 zones namely red zones (130 districts), orange zones (284 districts) and green zones (319 districts) with some relaxations in orange and green zones. Even then spread of COVID-19 is not under control, the lockdown is extended till 31 May 2020 with relaxation policies for the zones. Since the spread continues to grow exponentially in red zones, the lockdown is extended up to 30 June 2020 whereas other zones have been relaxed to restore the normal life with minimum restrictions. Any pandemic and localized epidemic is to critically go through five stages of eradication, invoking different stages [8], which at this point of writing India is still in Stage 3. Referring to Figure 1, it is important to tap the peak and to see the point at which the growth rate is still significantly less and raising where the recovery rate is faster, fatality rate is dropping, then Stage 3 is in place for the recovery phase. Eventually stage 2 is critical towards the containment of the pandemic of countries like Italy, Iran and US left out making the fatality rate very higher. However, in China, though there was a slack at Stage 1 they bounce back to push the Stage 2 with severe measures making it to contain faster[9]. 
India - Confirmed Cases

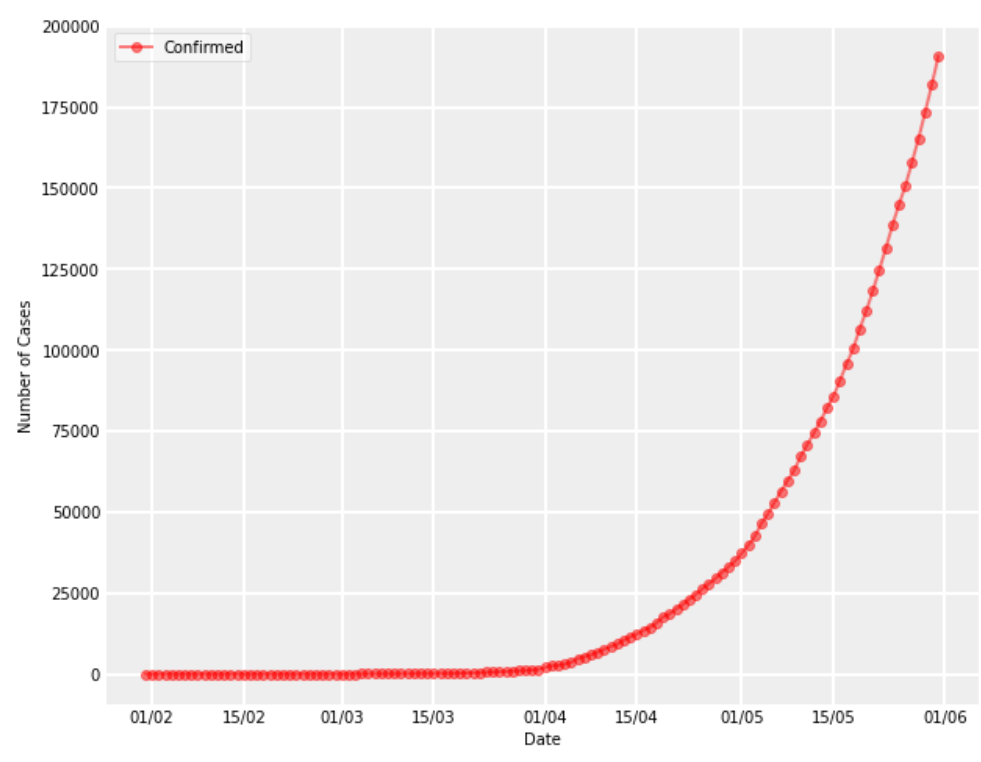

Figure 1. Confirmed Cases growth

Figure 2 shows the Chronological classification for the Wuhan case used in this study model. Stage 1(22-27 December 2020) is reported for the monitoring to weigh the class of severity of the outbreak. China reported a cluster of cases of pneumonia in Wuhan, Hubei Province (31 Dec 2019) (classified as epidemic) (Stage 2), From there several measures including the lockdown of the Hubei province makes the recovery picking up with downsizing the confirmed new cases (Stage3). The ratio of the growth rate of the outbreak is hitting low and recovery rate faster make the last Stage of restoration and subsequent opening the province for normalcy on the 9 April 2020. This is used onto the reference for the case of India as the population density is closer to that of the country but with the impetus on the Government putting strictest control at the start of the outbreak making the days for Stage 1 within control. However, the challenge lies in a country of fully democratic and more than three quarter lies below the poverty line, Stage 2 is where we believe the country will face difficulty, in contrast to China Government where the Communist control makes the adjustments faster. Hence, a profound model is critical to analyze and report critically which motivates us to present this work to the scientific community. 


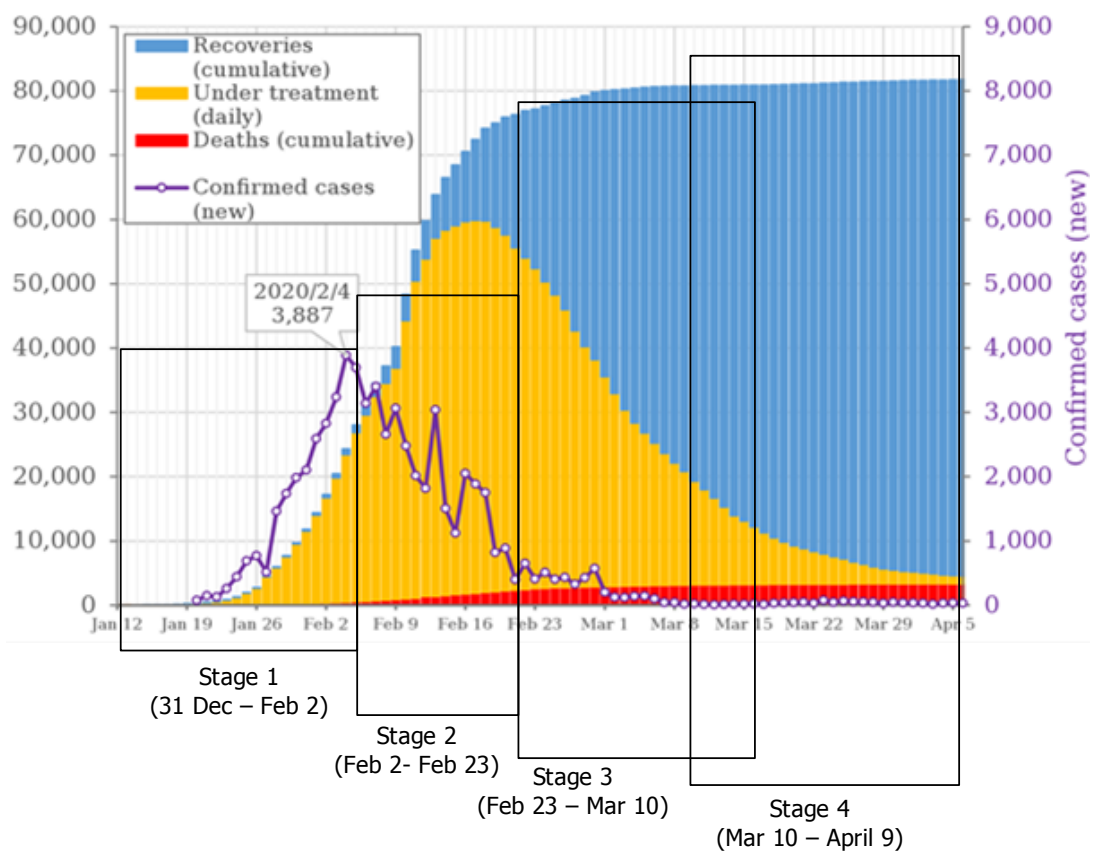

Figure 2 Chronological classification for the Wuhan case used in this study

(Picture Source: By Phoenix7777 - Own work Data source: 疫情通报 Outbreak notification, CC BY-SA 4.0, https://commons.wikimedia.org/w/index.php?curid=87342125)

At present, there are numerous studies focusing on the outbreak of Corona Virus. The contagion caused by corona virus belongs to the category of Susceptible-Infected-Removed (SIR) disease, where an infected person could die or recover and again contract the disease. The initial studies had their focus on the outbreak in the epicenter China and the predictions for it. SIR model had been used to study the spread of COVID-19 in China by constructing base model curves for the infected, recovered and dead. The behavior of the curve indicates that strategy adopted by China to contain the outbreak are satisfactory. The peak of the epidemic, according to the model, is found to be in midFebruary and currently, the recovery rate is increasing which is in accordance with already published works [10]. The mathematical model is very relevant to the current scenario in China thus emphasizing the accuracy of the model and it is an effective model to study the pandemic in other worst affected countries like Italy, France, USA and India as well.

\subsection{Methods}

\subsection{Simplified Model used for this study}

The SIR model had found wide applications in the study of Ebola epidemic, HIV epidemic, SARS and other diseases. To represent a model, the whole population with the number of suspected cases but which are not infected by the disease represented as 'S', infected cases as 'I' and diseased represented as ' $R$ ' [11]. The parameter $\beta$ represent 
infection rate at which an affected individual meets $\mathrm{N}$ number of other individuals who are not affected. It is shown as $\beta \mathrm{N}$, with the population size $\mathrm{N}$ fixed. The parameter $\gamma$ shows the recovery rate. It is assumed that the infected category is at a rate proportional to number of infected cases and number of suspected cases and the increase in the infected category is $\beta$ SI with $\beta>0$. It is also assumed that the rate of increase of recovered category that are moved from infected category is proportional to number of infected cases, $\gamma \mathrm{I}$, with $\gamma>0$ represented in Equation (1)-(3).

$$
\begin{aligned}
& \frac{\mathrm{ds}}{\mathrm{dt}}=-\beta \mathrm{SI} \\
& \frac{\mathrm{dI}}{\mathrm{dt}}=\beta \mathrm{SI}-\gamma \mathrm{I} \\
& \frac{\mathrm{d} r}{\mathrm{~d} t}=\gamma \mathrm{I}
\end{aligned}
$$

Initially, $\mathrm{S}(0)=S_{0}>0, I(0)=I_{0}>0, R(0)=0$. The population in susceptible case must be large for an epidemic to occur.

\subsection{Before Lockdown}

In this part of the work, the model is developed with fine tuning the infection rate by fitting the infected cases of real data with the simulated data. Due to low testing rate in the initial stage we choose t $0=10$ days after the first day (22/01/2020). Hence the time period considered is from $31 \mathrm{Jan} 2020$ to 22 March 2020 with $I_{0}=1$ and $R_{0}=0$. The infection rate $\beta_{t+1}$ is computed as per Equation (4).

$$
\beta_{t+1}=\left(I_{t+1}-I_{t}\right) / I_{t}
$$

Finally, the mean infection rate $\beta$ determined using Equation (5).

$$
\beta=\frac{\sum_{t=t_{0}}^{t_{n}} \beta(t)}{n}
$$

Similarly, the recovery rate for the time period $\gamma_{t+1}$ is found as per Equation (6). 
$\gamma_{t+1}=\left(R_{t+1}-R_{t}\right) / R_{t}$

The overall recovery rate $\gamma$ is determined as per Equation (7).

$\gamma=\frac{\sum_{t=t_{0}}^{t_{n}} \gamma(t)}{n}$
(6)

(7)

For a time period of 50 days, the values for $\beta=0.1232$ and $\gamma=0.0120$. The actual data is fitted against the infected cases as shown in Figure 3. For both infected and recovered cases, the actual data fits with minimal error. Based on the predictions of the model, if the infection rate remains as close as the present value the expected number of infected cases reach a maximum in the mid of April. However, it mainly depends on the number of effective cases monitored and quarantined to reduce the spread.

\section{Prediction BEFORE Lockdown (24/3/2020)}

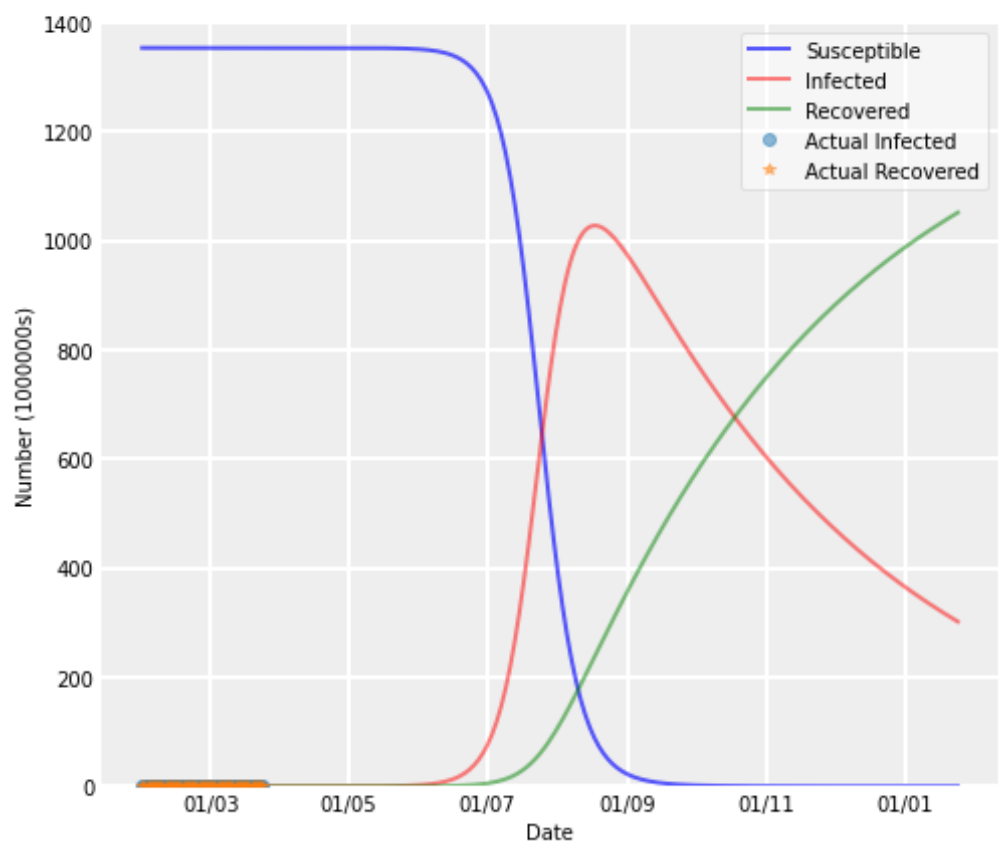

Figure 3. SIR model - Before Lockdown 


\subsection{After Lockdown}

Due to the immediate spread of the disease and vulnerability, Indian government has announced lockdown from 24 March 2020. While analyzing the data after the lockdown, the rate of growth of confirmed covid19 cases increased exponentially. The country moves to Stage 2 with exponential growth rate compared with other countries. Also, confirming the disease requires minimum 14 days. These factors cause the infection rate to considerably increase. Taking the data from 24 March 2020 to 17 May 2020, there is a considerable change in $\beta$ and $\gamma$. Hence the value obtained during this time period for $\beta$ and $\gamma$ are 0.1541 , and 0.0391 respectively. Figure 4 shows the SIR model predictions over four phases of the lockdown period (Period: 360 days). The prediction results (Figure 4a) considering Phase 1 [12] infected cases indicate that the rate of growth of COVID-19 infections tend to increase even though it was slowed down to prior lockdown thus leading to extend the lockdown (Phase 2). Figure 4b depict the effectiveness of containment strategies taken by the government [13] to reduce the infections compared to Phase 1 . It clearly helps to flatten the curve to some extent. Further reduction of spread requires Phase 3 lockdown [14] and the prediction results (Figure 4c) indicates that extension of the lockdown (Phase 4) with some relaxation polices [15]across different zones of the country is required. The simulation results depict that the number of infected cases increased in each phase than the earlier one. Also, recovery rate increased as few patients recovered from the disease after the minimum recovery period.

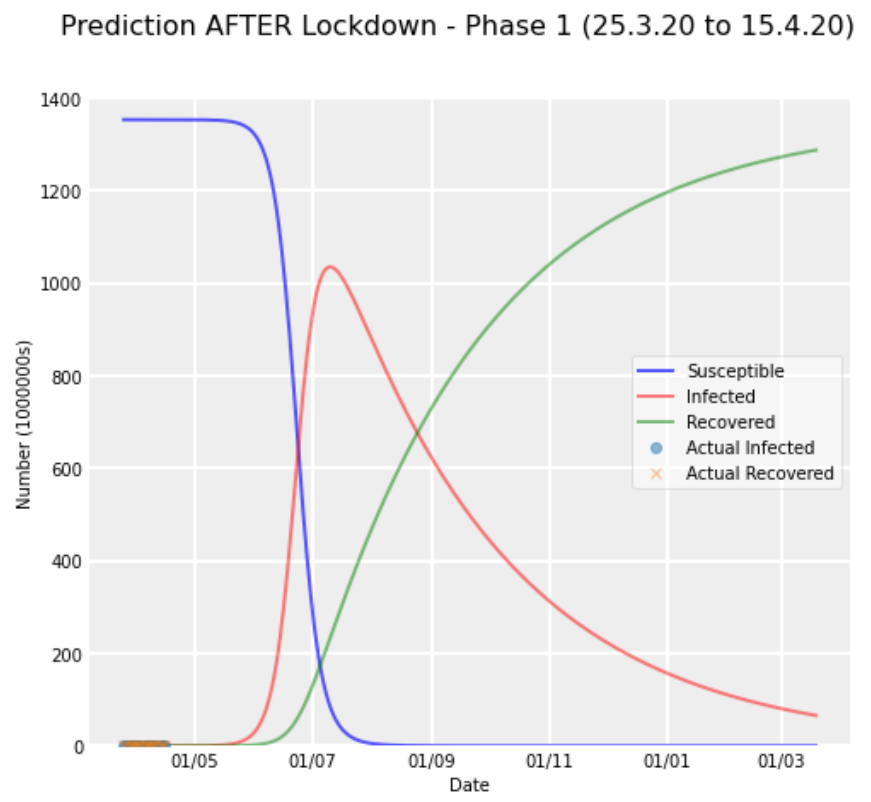

a. Phase 1 
Prediction AFTER Lockdown - Phase 2 (16.4.20 to 03.5.20)

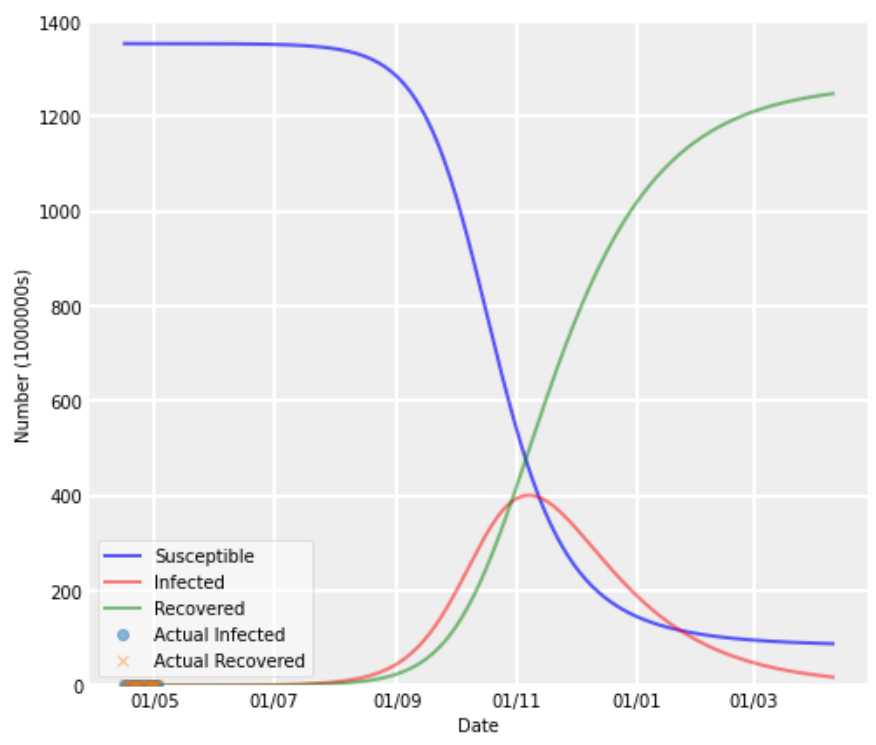

b. Phase 2

Prediction AFTER Lockdown - Phase 3 (4.5.20 to 17.5.20)

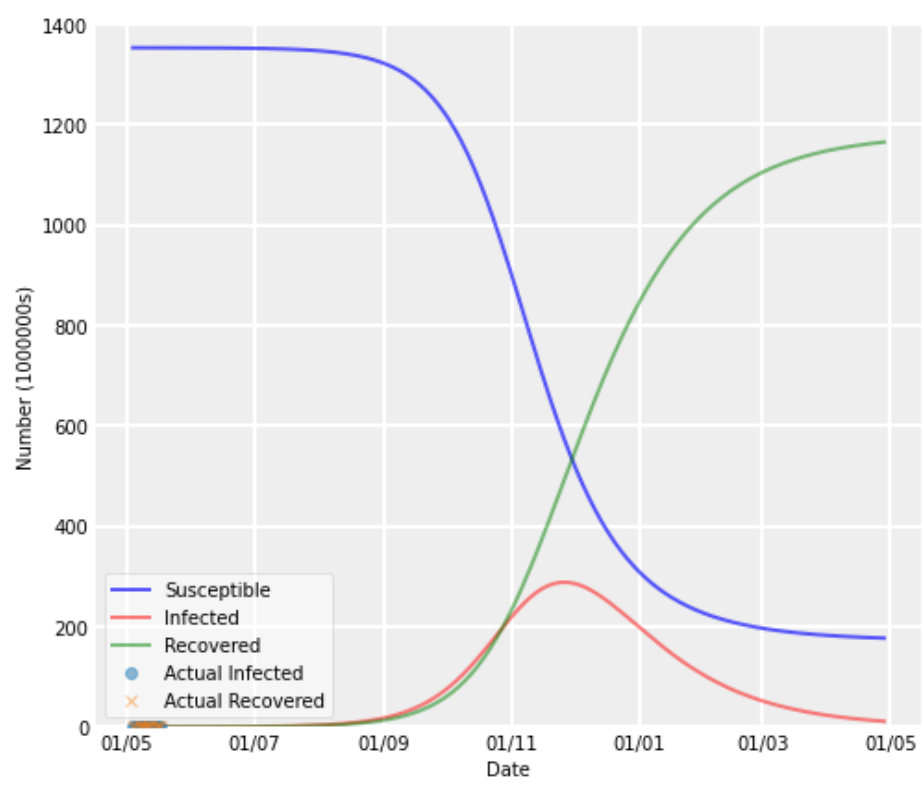

c. Phase 3 


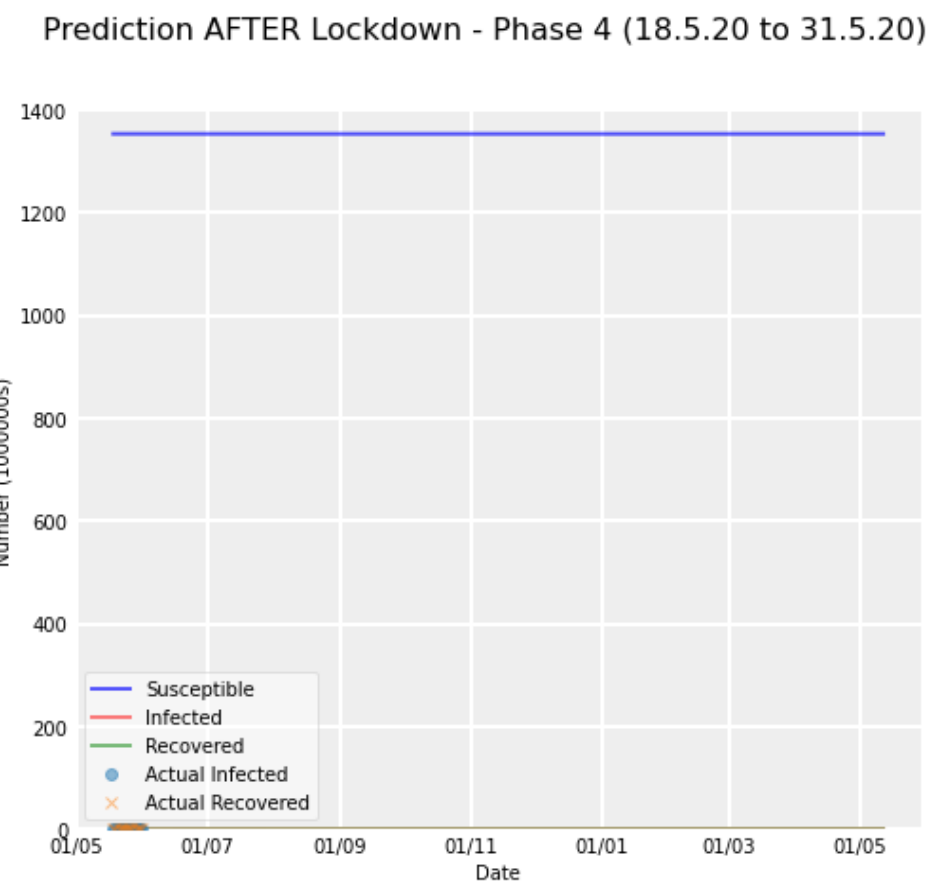

d. $\quad$ Phase 4

Figure 4. SIR model - After Lockdown with different Phases

\subsection{Integrated Model}

Figure 5 shows the model developed for the period 31 Jan 2020 to 31 May 2020. The infection rate and recovery rate fitted with the overall data are 0.18907 and 0.09396 respectively. While comparing the parameters with Model 2, the cumulative infection rate still increases due to effective testing measures. The recovery rate improved since certain number of infected cases recovered over the period. Since the lockdown causes to control the spread of the disease, it is noticed that it can be continued further to minimize the infection rate. The results of different runs with varying number of days for prediction depicts that the proposed model exhibits good fitness with actual data. 


\section{Prediction for Overall Data}

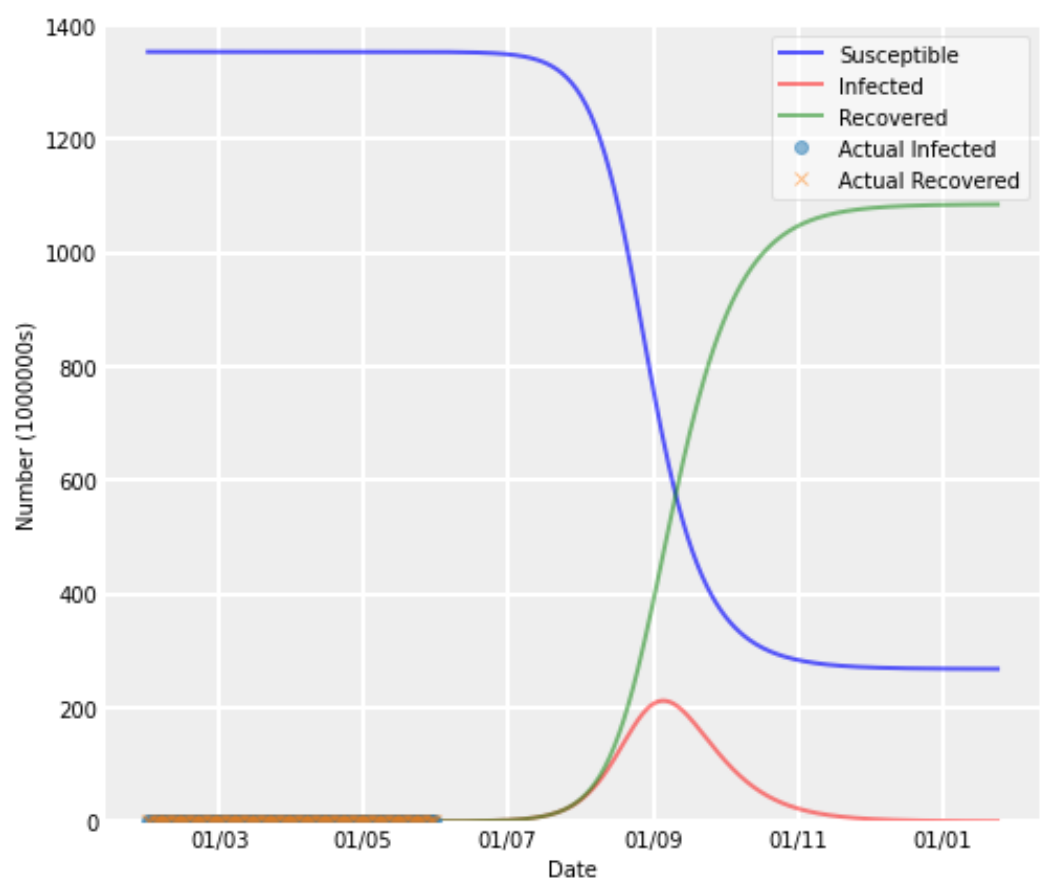

Figure 5. Integrated model - Overall data

Even though lock down enables to control the spread of the disease, the number of infections raised exponentially. Now, the government takes actions to identify the infected persons that leads to sudden increase in the number of cases. As India is now in stage 3, proper strategies are essential to continue social distancing among the people in order to prevent recurrence of the spread. The model forecasts that the number of cases will exponentially grow, and the inflection point can be reached with proper quarantine policies. When the number of people quarantined increases, there will be a decrease in the number of infected cases among the susceptible population. The adoption of quarantine policies by the susceptible population and public health measures, awareness methodologies help to decline the infection rate. The reduction also happens exponentially after reaching the inflection point where the number of infected cases is maximal. Thus, effective reduction of infections at different Quarantine rate (q) are examined in our model. While analysing the growth rate after lockdown, it exhibits $20 \%$ growth. Based on this, we examined the reduction of infection through the quarantine rate (q) with values of 20\%, 40\%, 60\% and $80 \%$ as seen in Figure 6. 


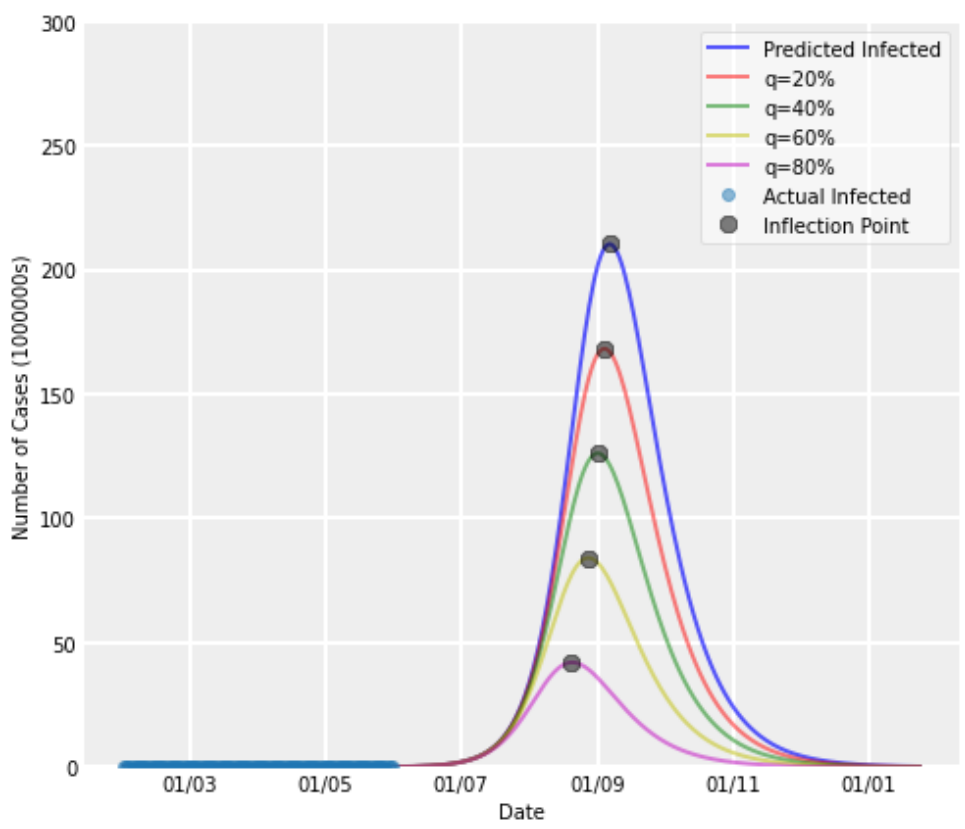

Figure 6. Effectivenes of Different Quarantine Rate(q)

The choice of the percentage is through the consideration on the growth rate of the infection and the rate at which the recovery happened. Figure 6 depicts the results of reduction in infection for different quarantine rates. The inflection point that reaches maximum number of cases are given in Table 1. The effective implementation of Quarantine measures $(80 \%)$ shall increase the infected cases at the maximum of 4,20,71,557 in the predicted model.

Table 1 Inflection point with varying Quarantine rate

\begin{tabular}{|l|l|l|l|}
\hline Quarantine rate (q) & Infection Point (Date) & Infected Cases & Recovery Rate \\
\hline $20 \%$ & $04 / 09 / 2020$ & 168305371 & $26.8 \%$ \\
\hline $40 \%$ & $01 / 09 / 2020$ & 126228155 & $33.2 \%$ \\
\hline $60 \%$ & $28 / 08 / 2020$ & 84135339 & $48.8 \%$ \\
\hline $80 \%$ & $20 / 08 / 2020$ & 42071557 & $100 \%$ \\
\hline
\end{tabular}

This profound model is critical to analyze and report critically which motivates us to present this work to the scientific community. The proposed model predicts the future cases for the data collected up to 31 May 2020. In the best case ( $q=60 \%$ ), prediction results enforce to continue the lockdown till 3 Sep 2020 since a minimum of 14 days is required to settle down after the inflection point on 20 August 2020. In the worst case for $\mathrm{q}=20 \%$ the maximum number of 
infected cases alone is $16,83,05,371$ with inflection point at 4 Sep 2020. For this scenario, the lock down must be extended up to 18 Sep 2020. This will help the government to streamline the disease spread with effective utilization of the existing resources to treat the disease. Figure 8 depicts the inflection dates of the disease spread for different quarantine rates.

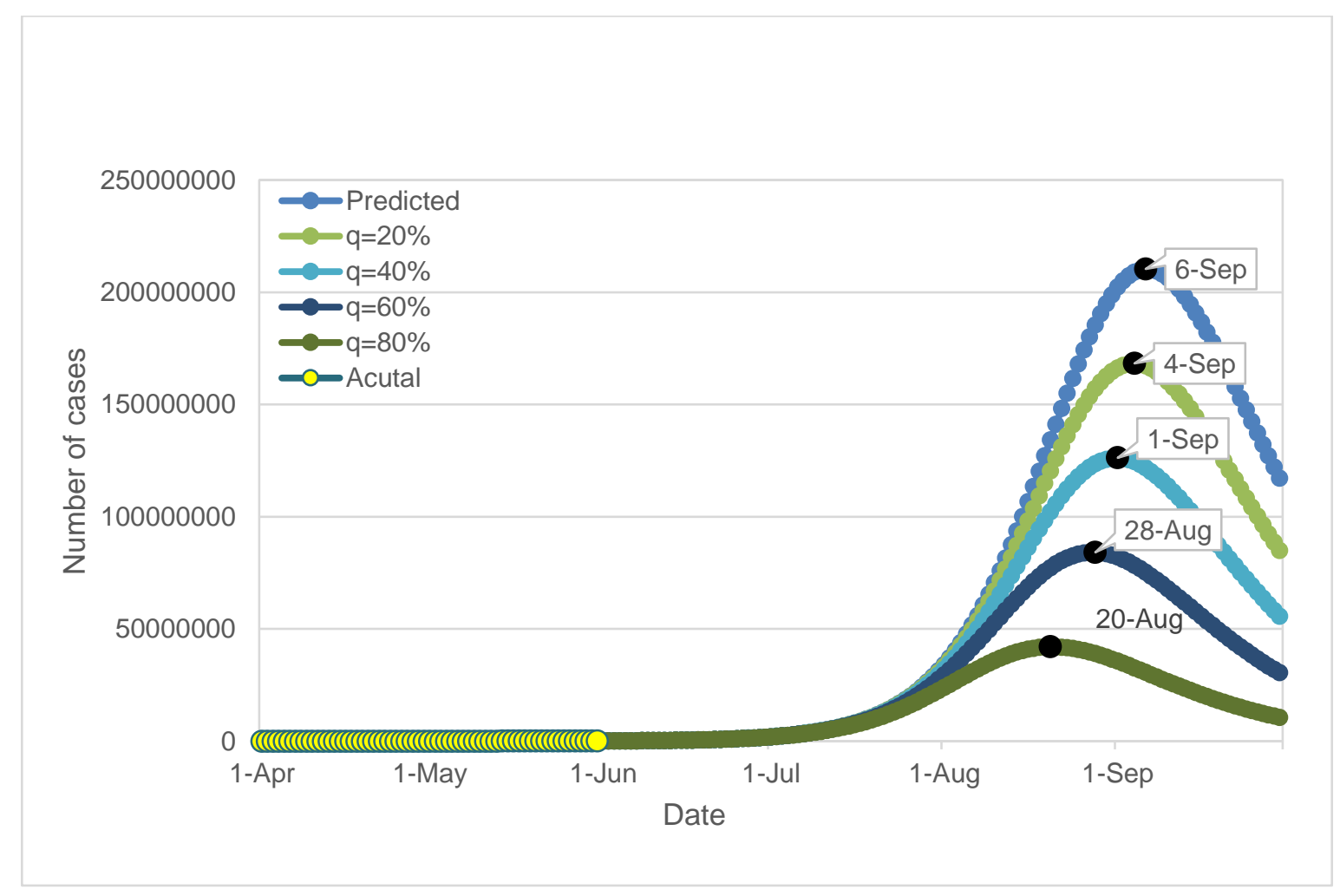

Figure 8. Inflection Dates of Covid-19 in India

This model is built from January and aims to predict the disease transmission rate in the forthcoming months. The model has taken the population of India. In the period after locking down, the numbers had been constantly increasing due to community transmission and adequate testing than the earlier scenario. A look at the testing statistics reveal that in India, 32 tests are performed for every million whereas Italy conducts 6524 tests per million, UK goes for 1714 tests per million and US 1916 per million. While at the initial stage, the rate of infection was minimal and during the transmission stage, the susceptible population migrates to the infected, the rate is higher. Prediction results indicate that till August 2020, social distancing shall be practiced bringing down the growth rate at the minimum and prevent reoccurrence of the outbreak. 


\section{Conclusions}

The impact of Covid-19 growth rate in India is lower than other countries across the world. A simplified model in this work forecast the maximum number of infections likely to occur by 6 Sep 2020 as per the current growth rate.

The model also identifies the different stages of the outbreak in the country. Predictions of the model suggest implementing the containment measures to bringdown the infections with different quarantine rate. As per the model, the infection rate is likely to decline from end of August and reach minimum by September when the containment measures are implemented with $60 \%-80 \%$ quarantine rate. This helps to manage the epidemic and bring it under control in India.

\section{Funding}

This research did not receive any specific grant from funding agencies in the public, commercial, or not-forprofit sectors

\section{Conflict of Interest}

Authors declare that they have no conflict of interest.

\section{References}

[1] T.L. Kamikawa, M.G. Mikolajczyk, M. Kennedy, L. Zhong, P. Zhang, E.B. Setterington, D.E. Scott, E.C. Alocilja, Pandemic influenza detection by electrically active magnetic nanoparticles and surface plasmon resonance, IEEE Trans. Nanotechnol. 11 (2012) 88-96. doi:10.1109/TNANO.2011.2157936.

[2] K. On, A. Tang, V.W.I. Wei, W. Hyun, E. Kiong, S. Riley, Epidemic Models ofContact Tracing: Systematic Review of Transmission Studies of Severe Acute Respiratory Syndrome and Middle East Respiratory Syndrome, 17 (2019) 186-194.

[3] WHO, Wuhan Municipal Health Commission Infection Data, (2020). http://wjw.wuhan.gov.cn/front/web/list2nd/no/710 (accessed April 2, 2020).

[4] NYT, New York Times, (2020). https://www.nytimes.com/2020/03/17/health/coronavirus-surfacesaerosols.html (accessed April 2, 2020).

[5] JHU, CSSEGISandData/COVID-19: Novel Coronavirus (COVID-19) Cases, provided by JHU CSSE, (n.d.). https://github.com/CSSEGISandData/COVID-19 (accessed June 9, 2020).

[6] ET, Economic times, (2020). https://economictimes.indiatimes.com/news/politics-and-nation/coronaviruscases-in-india-live-news-latest-updates-april10/liveblog/75074919.cms (accessed April 12, 2020).

[7] WHO, World Health Organization. (May 6, 2003). WHO The Cumulative Number of Reported Probable Cases of Severe Acute Respiratory Syndrome (SARS). Geneva, Swiss., (2003). https://www.who.int/csr/sarscountry/2003_05_06/en/ (accessed April 7, 2020).

[8] GT, Github, (2020). https://github.com/datasets/covid-19 (accessed April 12, 2020).

[9] CDC, CDC, (2020). https://www.cdc.gov/coronavirus/2019-ncov/symptoms-testing/symptoms.html (accessed April 12, 2020). 
[10] P. V Khrapov, A.A. Loginova, Mathematical modelling of the dynamics of the Coronavirus COVID-19 epidemic development in China, 8 (2020).

[11] Sulsky D, SIR Model, (2012). math.unm.edu (accessed April 12, 2020).

[12] NYT, India, Day 1: World's Largest Coronavirus Lockdown Begins - The New York Times, (n.d.). https://www.nytimes.com/2020/03/25/world/asia/india-lockdown-coronavirus.html (accessed May 20, 2020).

[13] IT, In coronavirus lockdown extension, Modi wields stick, offers carrot on exit route - Coronavirus Outbreak News, (n.d.). https://www.indiatoday.in/coronavirus-outbreak/story/in-coronavirus-lockdownextension-modi-wields-stick-offers-carrot-on-exit-route-1666741-2020-04-14 (accessed May 20, 2020).

[14] ET, India Lockdown Extension News: Lockdown extended by 2 weeks, India split into red, green and orange zones - The Economic Times, (n.d.). https://economictimes.indiatimes.com/news/politics-andnation/govt-extends-lockdown-by-two-weeks-permits-considerable-relaxations-in-green-and-orangezones/articleshow/75491935.cms (accessed May 20, 2020).

[15] ET, Lockdown 4.0 new guidelines: Lockdown 4.0 guidelines: Nationwide lockdown extended till May 31, with considerable relaxations - The Economic Times, (n.d.).

https://economictimes.indiatimes.com/news/politics-and-nation/centre-extends-nationwide-lockdown-tillmay-31-with-considerable-relaxations/articleshow/75790821.cms (accessed May 20, 2020). 